\title{
Review
}

\section{Stem Cells and Kidney Disease}

\author{
Keiichi HISHIKAWA ${ }^{1,2)}$ and Toshiro FUJITA ${ }^{1,2)}$
}

\begin{abstract}
Functional recovery in acute renal failure is well known, and the adult kidney is generally recognized to have the capacity to regenerate and repair. Several groups have reported the contribution of bone marrowderived cells in this process, and others have confirmed the existence of adult stem cells in the kidney, including slow-cycling cells, side population cells, CD133 + cells and rKS56 cells. However, recent data demonstrated that in vivo differentiation of bone marrow-derived cells into renal tubular cells may not occur at all, or is at most a minor component of the repair process. Moreover, it is now generally accepted that stem cells and multipotent cells contribute to the regenerative process by producing protective and regenerative factors rather than by directly differentiating to replace damaged cells. Therefore, for clinical regenerative medicine in kidney disease, the focus of stem cell biology will shift from multiple differentiation of cells or cell-therapy to multiple functions of the cells, such as the production of bone morphologic protein-7 and other regenerative factors. (Hypertens Res 2006; 29: 745-749)
\end{abstract}

Key Words: stem cell, regenerative medicine, renal failure, cell therapy

\section{Introduction}

Adult stem cells or organ-specific stem cells were initially recognized in the hematopoietic system, skin, and intestinal epithelia, where self-renewal is apparent, but the cells are also present in organs that do not have rapid rates of cell turnover, such as the nervous system (1). We can find functional recovery in acute renal failure (ARF), and the adult kidney is recognized to have the capacity to regenerate and repair. However, it is still unclear whether this process is mediated by simple cellular proliferation in situ, or by bone marrowderived stem cells or adult/organ-specific stem cells. As distinct from ARF, successful reversal of chronic renal disease is limited. However, Fioretto et al. reported reversal of diabetic nephropathy (2) and Zeisberg et al. reported reversal of chronic renal injury (3). The important problem to be solved is whether so-called stem cells or stem cell systems are involved in these regenerative processes. Although the number of human kidney transplants has increased, the demand greatly exceeds organ availability (4). If we can control the function of stem cells in the kidney and reverse chronic renal failure, we will not need kidney transplants that require immunosuppressive regimens. Accordingly, this article will focus on stem cells in the kidney and their role in kidney disease.

\section{Stem Cells That May Contribute to Renal Regeneration}

\section{Bone Marrow-Derived Cells}

Two human studies have suggested that some bone marrowderived cells homing to the kidney could incorporate into the renal tubules. In the tubules of renal biopsy specimens from eight male patients transplanted with female kidneys, Poulsom et al. found Y-chromosome-positive tubular cells within the tubules that co-expressed epithelial markers (5). However, the proportion of Y-chromosome-positive tubular cells ranged from $1.8 \%$ to $20 \%$. Gupta et al. reported finding Ychromosome-positive tubular cells in renal biopsies taken from two men transplanted with female kidneys, but the positive cells made up less than $1 \%$ of the tubular cells examined (6). In an animal study, transplantation of purified mesenchy-

From the ${ }^{1)}$ Department of Clinical Renal Regeneration, Graduate School of Medicine and ${ }^{2}$ Division of Nephrology and Endocrinology, Department of Internal Medicine, University of Tokyo, Japan.

Address for Reprints: Keiichi Hishikawa, M.D., Ph.D., Division of Nephrology and Endocrinology, Department of Internal Medicine, The University of Tokyo Hospital, Hongo 7-3-1, Bunkyo-ku, Tokyo 113-8655, Japan. E-mail: hishikawa-tky@umin.ac.jp

Received April 19, 2006; Accepted in revised form July 7, 2006. 
mal stem cells (MSCs) expressing green fluorescent protein (GFP) into mice "in the absence of injury" resulted in the appearance of GFP-positive cells in several organs, including liver, lung, muscle and kidney (7). In the kidney, GFP-positive cells were sparsely distributed, being present in only 2 of 13 mice examined at a frequency of 5-10 cells per kidney section. In transient renal ischemia and reperfusion models, purified bone marrow cells transgenically expressing $\beta$ galactosidase were transplanted, and $\beta$-galactosidase-positive cells were found in tubular cells. The authors concluded that bone marrow cells had differentiated into, or fused with, tubular epithelial cells. On the other hand, Szczypka et al. failed to detect bone marrow-derived tubular cells in the kidney sections of female mice with folic acid-induced tubular injury that had been transplanted with male bone marrow (8). Duffield et al. failed to find examples of bone marrowderived renal tubular cells in two mouse models of bone marrow transplantation: ischemic renal injury of male-to-female transplant recipients, and GFP-transgenic bone marrow donation to wild-type recipients (9). As mentioned above, the conflicting results reported by different groups have generated uncertainty as to whether bone marrow cells actually become tubular cells in vivo. On the basis of most current data, it seems that even if adult bone marrow-derived cells retain the capacity to differentiate into, or fuse with, renal tubular cells, it is an uncommon event and does not have a predominant role in the tubule regenerative process (10).

\section{Adult Stem Cells in the Kidney}

As distinct from other tissues, such as bone marrow, it has been difficult to isolate or confirm the existence of stem cells in the kidney, although several studies have suggested the existence of stem cells in the adult renal interstitium.

\section{Slow-Cycling Cells in the Papilla}

Adult stem cells are considered to have a slow cycling time (11-13), and thus Oliver et al. tried to distinguish the cells in the kidney by measuring the retention of the nucleotide label bromodeoxyuridine (BrdU), which is incorporated into the DNA of cells during DNA synthesis (14). BrdU was administered to 3-day-old rat and mice pups. At this age, because nephrogenesis in rodents continues after birth, many cells in the kidney were probably dividing and thus could incorporate BrdU. After a chase period of at least 2 months, during which the multiple cell divisions required for kidney growth would have diluted the BrdU content of most cells, incorporation of BrdU was analyzed in the kidney tissue. Oliver et al. (14) found that only the interstitium of the renal papilla contained an abundant population of cells that retained a strong BrdU signal. They also found that the cells entered the cell cycle and the BrdU signal quickly disappeared from the papilla in transient real ischemia models. Moreover, the isolated renal papilla cells were multi-potent and displayed other characteristics of adult stem cells, and when they were injected directly into the renal cortex, the cells incorporated into the kidney parenchyma.

\section{Side Population Cells}

In 1996, Goodell et al. reported a new method of obtaining an enriched population of hematopoietic stem cells from adult bone marrow in a single step by Hoechst 33342 staining and FACS sorting (15). The isolated cells were called side population (SP) cells, and the SP phenotype can be used to purify a stem cell-rich fraction. The SP phenotype is determined by the BCRP1/ABCG2 gene, and enforced expression of BCRP1/ABCG2 prevents hematopoietic differentiation (16). To determine core genes comprising a stem cell genetic program, several comprehensive microarray studies have been performed $(17,18)$. However, the number of overlapping genes among the reports was limited, and BCRP1/ABCG2 was the only gene that was expressed in ES cells, hematopoietic stem cells and neurosphere cells (19). These results suggest that SP cells may play a role as adult stem cells. In fact, skeletal muscle SP cells (20) may differentiate into endothelial cells (21) and bone marrow-derived SP cells into cardiomyocytes, endothelial cells (22) and osteoblast precursors (23). Recently, Hishikawa et al. found that kidney SP cells differentiated into multiple lineages in the presence of leukemia inhibitory factor via kidney-specific cadherin 16 (24). Moreover, the function of kidney SP cells was found to be regulated by basic helix-loop-helix transcription factor MyoR, and the cells resided in the interstitial spaces of the kidney (25).

\section{CD133+ Cells in the Interstitium}

CD133 is a surface marker of endothelial progenitor cells, hematopoietic progenitor cells and neural stem cells. Bussolati et al. reported that CD133-positive cells in the interstitium of the adult human kidney have characteristics of stem cells (26). The cells expressed the early nephron developmental marker Pax2, as well as several markers typical of bone marrow stromal cells, but were negative for hematopoietic cell markers such as CD34 or CD45. By using different culture conditions, the authors indicated that CD133+ renal cells might be pluri-potent, having the capacity to differentiate into either type of tubular cells with the appropriate cues.

\section{rKS56 Cells}

In the developing kidney, there are two major distinct areas of cell proliferation, the nephrogenic zone in the outer cortex below the renal capsule and the area in the corticomedullary junction corresponding to the primitive S3 segment of the proximal tubule (27). Kitamura et al. dissected individual nephrons from adult rat kidneys, then separated them into segments and cultured them (28). Outgrowing cells were replated after limiting dilution so that each well contained a single cell. In this way, they were able to isolate the cell line showing the most potent growth, which they designated rKS56. rKS56 cells expressed immature cell makers relating 
to kidney development and mature tubular markers. The location of rKS56 cells in kidney tissue is unclear, but rKS56 cells possessed self-renewal and multi-plasticity, and differentiated into mature tubular cells defined by aquaporin 1,2 expression under different culture conditions.

With the exception of rKS56 cells, all of the above types of cells were found in the interstitium but it is still unclear whether these cells are completely different types of cells or merely the same cells isolated by different methods. Moreover, the multipotency of these cells was evaluated under different conditions, and thus further studies will be required to conclusively determine the most potent cells that contribute to kidney regeneration or cell therapy.

\section{Stem/Progenitor Cell Therapy}

\section{Therapeutic Potential of Bone Marrow-Derived Cells}

As mentioned above, there are conflicting results as to whether bone marrow-derived cells play a predominant role in the tubule regenerative process. However, several studies have suggested the therapeutic potential of these cells in treating renal failure. Infusion of $5 \times 10^{5}$ lineage-negative cells at the time of ischemia-reperfusion resulted in partial improvement (29), but significant protection has been demonstrated when between $2 \times 10^{5}$ and $5 \times 10^{5}$ purified MSCs were infused $(9,30)$. On the other hand, several groups have shown that the mobilization of endogenous bone marrow-derived cells is a therapeutic option for ARF. Togel et al. found that G-CSF with cyclophosphamide that was used to mobilize hematopoietic stem cells (HSCs) worsened the progression of renal failure (31), but two groups have found that HSC mobilization with either stem cell factor (SCF) plus G-CSF protects mice from $\operatorname{ARF}(32,33)$. Iwasaki et al. reported that GCSF plus M-CSF accelerated the drop in blood urea nitrogen (BUN) and creatinine 4 days after cisplatin injection (33). GCSF may directly affect cell types other than bone marrowderived cells, and it is not clear whether these regimens mobilize bone marrow-derived cells in addition to HSCs, or whether MSCs are physiologically mobilized to exit the bone marrow in significant numbers under any conditions. At present, it seems that the bone marrow probably harbors a population of poorly understood cells such as endothelial progenitor cells (34) that has a protective effect in animal models of ARF when infused in large numbers.

\section{Therapeutic Potential of Adult Stem Cells in the Kidney}

Infusion/transplantation of adult stem cells into the kidney, such as slow-cycling cells, kidney SP cells, and rKS56 cells, has been shown to augment the recovery from ARF. And while this suggests that adult stem cells could be used for the treatment of ARF, it would probably be difficult to reverse chronic renal failure (CRF) by infusion/transplantation of these cells. Because these cells differentiate into multiple lineages, it may be theoretically possible to induce these cells to kidney precursors in vitro. Rogers et al. $(35,36)$ and Rogers and Hammerman $(37,38)$. reported that transplants of murine precursors to the adult kidney could be a potential source for regenerating kidney cells, and a promising solution for the current shortage of organs for kidney transplantation. Moreover, Dekel et al. demonstrated that human kidney precursors transplanted into mice differentiate into functional nephrons and not into other, non-renal cell types (4). The existence of slow-cycling cells and rKS56 cell has not been confirmed in humans, but CD133-positive cells and SP cells (Imai et al., under submission) exist in human kidney tissue. Taken together, these results suggest that making kidney precursors from adult stem cells in the kidney obtained from patients with CRF is worth trying, and transplantation of such precursors would be an ideal transplantation therapy without rejection.

\section{Activation of Adult Stem Cells in the Kidney}

The efficacy of cell therapy using bone marrow-derived cells or adult stem cells has been confirmed in cases of ARF, but not in cases of chronic renal failure. However, activation of adult stem cells in situ may reverse chronic renal failure. It is generally accepted that repair of the tubules after injury is mediated by the surviving tubular cells. After the injury, these cells lose their brush borders and dedifferentiate into a more mesenchymal type. These cells proliferate and eventually redifferentiate into an epithelial phenotype, completing the repair process. In this process, it is thought that the local release of growth factors such as hepatocyte growth factor (HGF) (39), epidermal growth factor, and insulin-like growth factor-1 coordinate this process of differentiation, migration, proliferation, and eventually re-differentiation (40). Zeisberg et al. showed that, unlike the above factors, bone morphologic protein (BMP)-7 reverses the transforming growth factor (TGF)- $\beta 1$-induced epithelial to mesenchymal transition by induction of E-cadherin (3). Moreover, they demonstrated that recombinant human BMP-7 leads to repair of severely damaged tubular epithelial cells, in association with reversal of chronic renal injury. These results strongly suggest that BMP-7 could be a potential therapeutic agent for chronic renal disease, but its gene expression is retained in only a few types of adult tissue, most prominently in the kidney, and its physiologic functions and regulation in the adult kidney are unknown at present. Recently we showed that retinoic acid down-regulated MyoR, a basic helix-loop-helix protein, and augmented the expression of BMP-7 in kidney SP cells (25). We also found that trichostatin A (TSA) downregulated MyoR, and augmented expression of BMP-7 in kidney SP cells and reversed chronic renal dysfunction (41; Imai et al., under submission). These results indicate that SP cells play an 
important role in the kidney as generator cells of BMP-7, and TSA as a stimulator of the cells, in situ, in reversing chronic renal disease.

\section{Conclusion}

Recent studies have demonstrated that stem cells and multipotent cells contribute to the regenerative process by producing protective and regenerative factors rather than directly by differentiating to replace damaged cells (41-44). For clinical regenerative medicine in kidney disease, we believe the focus of stem cell biology may shift from multiple differentiation of cells or cell therapy to multiple functions of cells, such as the production of BMP-7, HGF and other regenerative factors.

\section{References}

1. Rosenthal N: Prometheus's vulture and the stem-cell promise. N Engl J Med 2003; 349: 267-274.

2. Fioretto P, Steffes MW, Sutherland DE, Goetz FC, Mauer M: Reversal of lesions of diabetic nephropathy after pancreas transplantation. $N$ Engl J Med 1998; 339: 69-75.

3. Zeisberg M, Hanai J, Sugimoto H, et al: BMP-7 counteracts TGF-betal-induced epithelial-to-mesenchymal transition and reverses chronic renal injury. Nat Med 2003; 9: 964968.

4. Dekel B, Burakova T, Arditti FD, et al: Human and porcine early kidney precursors as a new source for transplantation. Nat Med 2003; 9: 53-60.

5. Poulsom R, Forbes SJ, Hodivala-Dilke K, et al: Bone marrow contributes to renal parenchymal turnover and regeneration. J Pathol 2001; 195: 229-235.

6. Gupta S, Verfaillie C, Chmielewski D, Kim Y, Rosenberg ME: A role for extrarenal cells in the regeneration following acute renal failure. Kidney Int 2002; 62: 1285-1290.

7. Anjos-Afonso F, Siapati EK, Bonnet D: In vivo contribution of murine mesenchymal stem cells into multiple cell-types under minimal damage conditions. J Cell Sci 2004; 117: 5655-5664.

8. Szczypka MS, Westover AJ, Clouthier SG, Ferrara JL, Humes HD: Rare incorporation of bone marrow-derived cells into kidney after folic acid-induced injury. Stem Cells 2005; 23: 44-54.

9. Duffield JS, Park KM, Hsiao LL, et al: Restoration of tubular epithelial cells during repair of the postischemic kidney occurs independently of bone marrow-derived stem cells. $J$ Clin Invest 2005; 115: 1743-1755.

10. Krause D, Cantley LG: Bone marrow plasticity revisited: protection or differentiation in the kidney tubule? J Clin Invest 2005; 115: 1705-1708.

11. Cotsarelis G, Cheng SZ, Dong G, Sun TT, Lavker RM: Existence of slow-cycling limbal epithelial basal cells that can be preferentially stimulated to proliferate: implications on epithelial stem cells. Cell 1989; 57: 201-209.

12. Johansson CB, Momma S, Clarke DL, Risling M, Lendahl $\mathrm{U}$, Frisen J: Identification of a neural stem cell in the adult mammalian central nervous system. Cell 1999; 96: 25-34.

13. Lavker RM, Sun TT: Epidermal stem cells: properties markers and location. Proc Natl Acad Sci U S A 2000; 97: 13473-13475.

14. Oliver JA, Maarouf O, Cheema FH, Martens TP, Al-Awqati Q: The renal papilla is a niche for adult kidney stem cells. $J$ Clin Invest 2004; 114: 795-804.

15. Goodell MA, Brose K, Paradis G, Conner AS, Mulligan $\mathrm{RC}$ : Isolation and functional properties of murine hematopoietic stem cells that are replicating in vivo. J Exp Med 1996; 183: 1797-1806.

16. Zhou S, Schuetz JD, Bunting KD, et al: The ABC transporter Bcrp1/ABCG2 is expressed in a wide variety of stem cells and is a molecular determinant of the side-population phenotype. Nat Med 2001; 7: 1028-1034.

17. Ivanova NB, Dimos JT, Schaniel C, Hackney JA, Moore KA, Lemischka IR: A stem cell molecular signature. Science 2002; 298: 601-604.

18. Ramalho-Santos M, Yoon S, Matsuzaki Y, Mulligan RC, Melton DA: "Stemness": transcriptional profiling of embryonic and adult stem cells. Science 2002; 298: 597-600.

19. Easterday MC, Dougherty JD, Jackson RL, et al: Neural progenitor genes. Germinal zone expression and analysis of genetic overlap in stem cell populations. Dev Biol 2003; 264: 309-322.

20. Asakura A, Seale P, Girgis-Gabardo A, Rudnicki MA: Myogenic specification of side population cells in skeletal muscle. J Cell Biol 2002; 159: 123-134.

21. Majka SM, Jackson KA, Kienstra KA, Majesky MW, Goodell MA, Hirschi KK: Distinct progenitor populations in skeletal muscle are bone marrow derived and exhibit different cell fates during vascular regeneration. J Clin Invest 2003; 111: 71-79.

22. Jackson KA, Majka SM, Wang H, et al: Regeneration of ischemic cardiac muscle and vascular endothelium by adult stem cells. J Clin Invest 2001; 107: 1395-1402.

23. Olmsted-Davis EA, Gugala Z, Camargo F, et al: Primitive adult hematopoietic stem cells can function as osteoblast precursors. Proc Natl Acad Sci US A 2003; 100: $15877-$ 15882.

24. Hishikawa K, Marumo T, Miura S, et al: Leukemia inhibitory factor induces multi-lineage differentiation of adult stem-like cells in kidney via kidney-specific cadherin 16 . Biochem Biophys Res Commun 2005; 328: 288-291.

25. Hishikawa K, Marumo T, Miura S, et al: Musculin/MyoR is expressed in kidney side population cells and can regulate their function. J Cell Biol 2005; 169: 921-928.

26. Bussolati B, Bruno S, Grange $\mathrm{C}$, et al: Isolation of renal progenitor cells from adult human kidney. Am J Pathol 2005; 166: 545-555.

27. Cha JH, Kim YH, Jung JY, Han KH, Madsen KM, Kim J: Cell proliferation in the loop of Henle in the developing rat kidney. J Am Soc Nephrol 2001; 12: 1410-1421.

28. Kitamura S, Yamasaki Y, Kinomura M, et al: Establishment and characterization of renal progenitor like cells from S3 segment of nephron in rat adult kidney. FASEB J 2005; 19: 1789-1797.

29. Kale S, Karihaloo A, Clark PR, Kashgarian M, Krause DS, Cantley LG: Bone marrow stem cells contribute to repair of the ischemically injured renal tubule. J Clin Invest 2003; 112: 42-49.

30. Morigi M, Imberti B, Zoja C, et al: Mesenchymal stem cells 
are renotropic helping to repair the kidney and improve function in acute renal failure. $J$ Am Soc Nephrol 2004; 15: 1794-1804.

31. Togel F, Isaac J, Westenfelder C: Hematopoietic stem cell mobilization-associated granulocytosis severely worsens acute renal failure. J Am Soc Nephrol 2004; 15: 1261-1267.

32. Stokman G, Leemans JC, Claessen N, Weening JJ, Florquin S: Hematopoietic stem cell mobilization therapy accelerates recovery of renal function independent of stem cell contribution. J Am Soc Nephrol 2005; 16: 1684-1692.

33. Iwasaki M, Adachi Y, Minamino K, et al: Mobilization of bone marrow cells by G-CSF rescues mice from cisplatininduced renal failure and M-CSF enhances the effects of GCSF. J Am Soc Nephrol 2005; 16: 658-666.

34. Imanishi T, Kobayashi K, Hano T, Nishio I: Effect of estrogen on differentiation and senescence in endothelial progenitor cells derived from bone marrow in spontaneously hypertensive rats. Hypertens Res 2005; 28: 763-772.

35. Rogers SA, Lowell JA, Hammerman NA, Hammerman MR: Transplantation of developing metanephroi into adult rats. Kidney Int 1998; 54: 27-37.

36. Rogers SA, Liapis H, Hammerman MR: Transplantation of metanephroi across the major histocompatibility complex in rats. Am J Physiol Regul Integr Comp Physiol 2001: 280: R132-R136.

37. Rogers SA, Hammerman MR: Transplantation of rat meta- nephroi into mice. Am J Physiol Regul Integr Comp Physiol 2001; 280: R1865-R1869.

38. Rogers SA, Hammerman MR Transplantation of metanephroi after preservation in vitro. Am J Physiol Regul Integr Comp Physiol 2001; 281: R661-R665.

39. Yamamoto Y, Kohara K, Tabara Y, Igase M, Nakura J, Miki T: Plasma hepatocyte growth factor and the relationship between risk factors and carotid atherosclerosis. Hypertens Res 2002; 25: 661-667.

40. Schena FP: Role of growth factors in acute renal failure. Kidney Int Suppl 1998; 66: S11-S15.

41. Hishikawa K: Do we really need "stem" cell for renal regeneration? Kidney Int 2005; 68: 1966-1967.

42. Murry CE, Soonpaa MH, Reinecke H, et al: Haematopoietic stem cells do not transdifferentiate into cardiac myocytes in myocardial infarcts. Nature 2004; 428: 664-668.

43. Balsam LB, Wagers AJ, Christensen JL, Kofidis T, Weissman IL, Robbins RC: Haematopoietic stem cells adopt mature haematopoietic fates in ischaemic myocardium. Nature 2004; 428: 668-673.

44. Uchimura H, Marumo T, Takase O, et al: Intrarenal injection of bone marrow-derived angiogenic cells reduces endothelial injury and mesangial cell activation in experimental glomerulonephritis. J Am Soc Nephrol 2005; 16: 997-1004. 